\title{
A concepção de epistemologia da organização do conhecimento
}

The conception of epistemology of knowledge organization

\author{
Paula Carina de Araújo \\ Universidade Federal do Paraná. Departamento de Ciência \\ $e$ Gestão da Informação / Universidade do Estado de Santa \\ Catarina. Programa de Pós-graduação em Gestão da \\ Informação, Brasil \\ paulacarina@ufpr.br \\ (D) https://orcid.org/0000-0003-4608-752X
}

\section{José Augusto Chaves Guimaräes}

Universidade Estadual Paulista "Júlio de Mesquita Filho".

Departamento de Ciência da Informação, Brasil

guima@marilia.unesp.br

(iD https://orcid.org/0000-0002-0310-2331

\author{
Joseph T Tennis \\ University of Washington (UW). The Information School, \\ Estados Unidos \\ jtennis@uw.edu \\ (iD https://orcid.org/0000-0003-1506-2549
}

\section{Resumo:}

Objetivo: Estudo sobre posições epistêmicas que influenciam o domínio da organização do conhecimento a partir da análise da literatura científica. Objetiva descrever a concepção de epistemologia no periódico Knowledge Organization por meio da metateoria.

Método: Pesquisa exploratória e descritiva que desenvolve um estudo metateórico baseado na metateoria de Ritzer. Duas ferramentas da teoria fundamentada em dados foram utilizadas, codificação e memorando.

Resultado: Três famílias de atributos foram identificadas a partir do processo de codificação e do memorando: o conceito de epistemologia na organização do conhecimento, seu propósito e as posições epistêmicas que influenciam o domínio. A terceira família de atributos é analisada neste artigo. Empirismo, racionalismo, historicismo e pragmatismo são descritos como as principais posiçôes epistêmicas que influenciam o domínio. O pragmatismo predomina na análise, seguido das teorias críticas e do historicismo.

Conclusões: $\mathrm{O}$ estudo conclui que a concepção da epistemologia no domínio da organização do conhecimento é o estudo crítico dos princípios, hipóteses e produção de conhecimento no domínio. A epistemologia se preocupa com o conhecimento científico produzido pelo domínio da organização do conhecimento, bem como com a aplicação do conhecimento para a criação dos sistemas de organização do conhecimento para apoiar o processo de organização do conhecimento como um todo.

Palavras-chave: Organização do conhecimento, Epistemologia, Posição epistêmica, Metateoria, Teoria Fundamentada em Dados.

\section{Abstract:}

Objective: Study about the epistemic stances that influence the knowledge organization domain by analyzing its scholarly literature. It aims to describe the conception of epistemology in the journal Knowledge Organization through metatheory.

Methods: It is an exploratory and descriptive research that develops a metatheoretical study based on Ritzer's metatheory. In the analysis stage, two grounded theory tools were used, coding and memoing. 
Results: Three attribute families arose from the coding and memoing processes: the concept of epistemology in knowledge organization, the purpose of epistemology of knowledge organization and, epistemic stances influencing the knowledge organization domain. The third attribute family is analyzed in this paper. Empiricism, rationalism, historicism and pragmatism are described as the mains epistemic stances influencing the domain. The pragmatist stance is prominent in the analysis, followed by critical theories and historicism.

Conclusions: The study concludes that the conception of epistemology on the knowledge organization domain is the critical study of principles, hypothesis and knowledge production in the domain. Epistemology is concerned with the scientific knowledge produced by the knowledge organization domain, as well as with the application of that knowledge to design the Knowledge Organization Systems and to support the knowledge organization process as a whole.

KEYWORDS: Knowledge organization, Epistemology, Epistemic Stance, Metatheory. Grounded Theory.

\section{INTRODUÇÃo}

A organização do conhecimento (OC) é tanto a atividade de ordenar e representar informações quanto o campo de estudo que se preocupa com a natureza e a qualidade dos processos e sistemas de OC (Hjørland, 2003, 2008, 2013b; Tennis, 2008).

Para os fins deste artigo, OC é o campo de estudo que se preocupa com a natureza e a qualidade do processo de organização e representação do conhecimento, uma vez que esta pesquisa trata da epistemologia da OC. Os estudos epistemológicos são considerados uma das onze abordagens propostas por Hjørland (2002b; 2017) no domínio da OC e são relacionados a pelo menos dois propósitos. Primeiro, reconhecer como diferentes posturas epistêmicas influenciam a prática de representar o conhecimento. Segundo, entender melhor os fundamentos, teorias e métodos que influenciam a pesquisa no domínio.

Os estudos que buscam compreender os fundamentos, teorias e métodos que influenciam a pesquisa no domínio da OC são, em sua maioria, estudos teóricos. Muitos deles usam metateoria, implícita ou explicitamente, para obter melhor entendimento do domínio, para ter uma perspectiva completa e, também, para produzir nova teoria, (Dousa, 2010; Hjørland, 2002a, 2005, 2014; Tennis, 2005a; 2008; 2015). Esta pesquisa utiliza a metateoria como método.

Nesse contexto, as questões de pesquisa deste estudo é: qual é a concepção de epistemologia no domínio da OC? E, quais epistemologias influenciam a pesquisa no domínio da OC? As posturas epistêmicas influenciam tanto as questões relacionadas à representação do conhecimento (critérios de relevância, necessidades de informação, classificação, indexação, recuperação de informação, etc.) quanto a pesquisa em OC. Portanto, o objetivo geral deste estudo é descrever a concepção de epistemologia no domínio da OC por meio da metateoria. Portanto, são apresentados os seguintes objetivos específicos:

a) analisar a literatura científica sobre epistemologia da organização do conhecimento publicada no periódico Knowledge organization;

b) apresentar um compreensão profunda das principais epistemologias que influenciam o domínio da OC

A Knowledge organization é uma publicação que representa o pensamento da área, por ser considerada a revista científica referência do domínio pela International Society for Knowledge Organization (ISKO) (ISKO, 2019). Por isso, o corpus desta pesquisa é formado por artigos publicados no periódico Knowledge organization.

"Contribuições teóricas genuínas para a OC são muito raras, mas parecem obrigatórias em relação aos desafios com os quais este campo se confronta" (Hjørland, 2008, p. 87). O autor fez essa afirmação ao discutir abordagens teóricas para a $\mathrm{OC}$ e, esse é um forte argumento em relação à importância deste estudo para o domínio.

A motivação para desenvolver a investigação proposta neste artigo surgiu do reconhecimento do segundo propósito dos estudos epistemológicos, para melhor compreender os fundamentos, teorias e métodos 
que influenciam a investigação na área. "São as interações das prioridades ontológicas, epistemológicas e sociológicas que definem o trabalho de um domínio como atividade produtiva e, assim, revela seu papel crítico tanto na evolução do conhecimento quanto na compreensão do conhecimento como entidade científica" (López-Huertas, 2015, p. 578). Importa saber como a epistemologia influencia o trabalho teórico no domínio da OC e também qual é seu impacto no mundo social.

Acredita-se que a literatura científica, seja qual for seu grau de desenvolvimento ou sua formalização metodológica, sempre implica em modos de consciência pelos quais a pesquisa dá sentido à sua prática (Bufrem, 2012). Reforça-se ainda a "existência de uma preocupação da área em resgatar sua base epistemológica, seus traços conceituais, suas influências teóricas, seus matizes metodológicos, enfim, sua essência enquanto área de estudos" (Guimarães, 2014, p. 15).

Esta introdução é seguida pela descrição da metodologia. A seção 3 apresenta a síntese metateórica por meio da discussão da concepção de epistemologia do domínio da OC. Por fim, as considerações finais são apresentadas na seção 4 .

\section{Metodologia}

Desenvolve-se uma pesquisa exploratória e descritiva, pois os dados são analisados e descritos buscando apresentar uma metateoria da epistemologia da OC. A pesquisa também caracteriza-se como uma investigação metateórica baseada na metateoria de Ritzer (1991b, 2001).

Metateoria é um trabalho meta-analítico que surgiu da sociologia (Ritzer, 1991a, p. 237, tradução nossa) e é descrita como "o conjunto de suposições pressupostas por qualquer corpo de afirmações mais ou menos formalizado" (Bullock, 1989). Pode-se dizer que um conceito em uma metateoria consiste em uma gama de variações de significados fixados pela definição do conceito". Portanto, a metateoria apoia a análise e a compreensão da concepção de um conceito em um domínio.

Ritzer (2001, p. 15, tradução nossa) explica que a "metateoria, [...], não é um processo que ocorre antes de a teoria ser desenvolvida para estabelecer seus pré-requisitos. Em vez disso, o desenvolvimento de uma metateoria é um processo que ocorre após a criação da teoria e toma essa teoria como objeto de estudo".

A metateoria de um domínio pode ser comparada à análise de seus pressupostos. Seguindo esse raciocínio, Vickery (1998) aponta que para alcançar o sucesso por meio da metateoria, é importante se conectar com os pressupostos já existentes no domínio, mostrar as fragilidades e propor alternativas.

Ritzer (1991b) diferencia entre três tipos de metateoria: metateoria para melhor compreender um domínio $(\mathrm{Mu})$, como um prelúdio para a produção de uma nova teoria $(\mathrm{Mp})$ e para produzir uma perspectiva que ultrapassa alguma parte ou todo o domínio (Mo).

A aplicação da metateoria para melhor compreender um domínio $(\mathrm{Mu})$ tem duas dimensões: internaexterna e intelectual-social. As dimensões "são contínuas sem linhas duras e rápidas entre os polos de cada um” (Ritzer, 1991b, p. 17, tradução nossa). Pode-se explicar as duas dimensões, seguindo o pensamento de Ritzer (1991b, p. 17, tradução nossa):

Interno: relacionado a elementos que existem dentro do domínio.

Externo: refere-se a fenômenos que são encontrados fora do domínio, mas que têm impacto sobre ele.

Intelectual: teorias, ferramentas metateóricas, ideias emprestadas de outras disciplinas, com alguma relação com a estrutura cognitiva do domínio.

Social: lida com a estrutura sociológica do domínio: escolas, o efeito de fatores individuais de fundo, o impacto da sociedade em geral.

A combinação das dimensões interna-externa e intelectual-social gera uma tabela com quatro abordagens que expressam os quatro tipos de $\mathrm{Mu}$, como pode ser observado na Figura 1. 


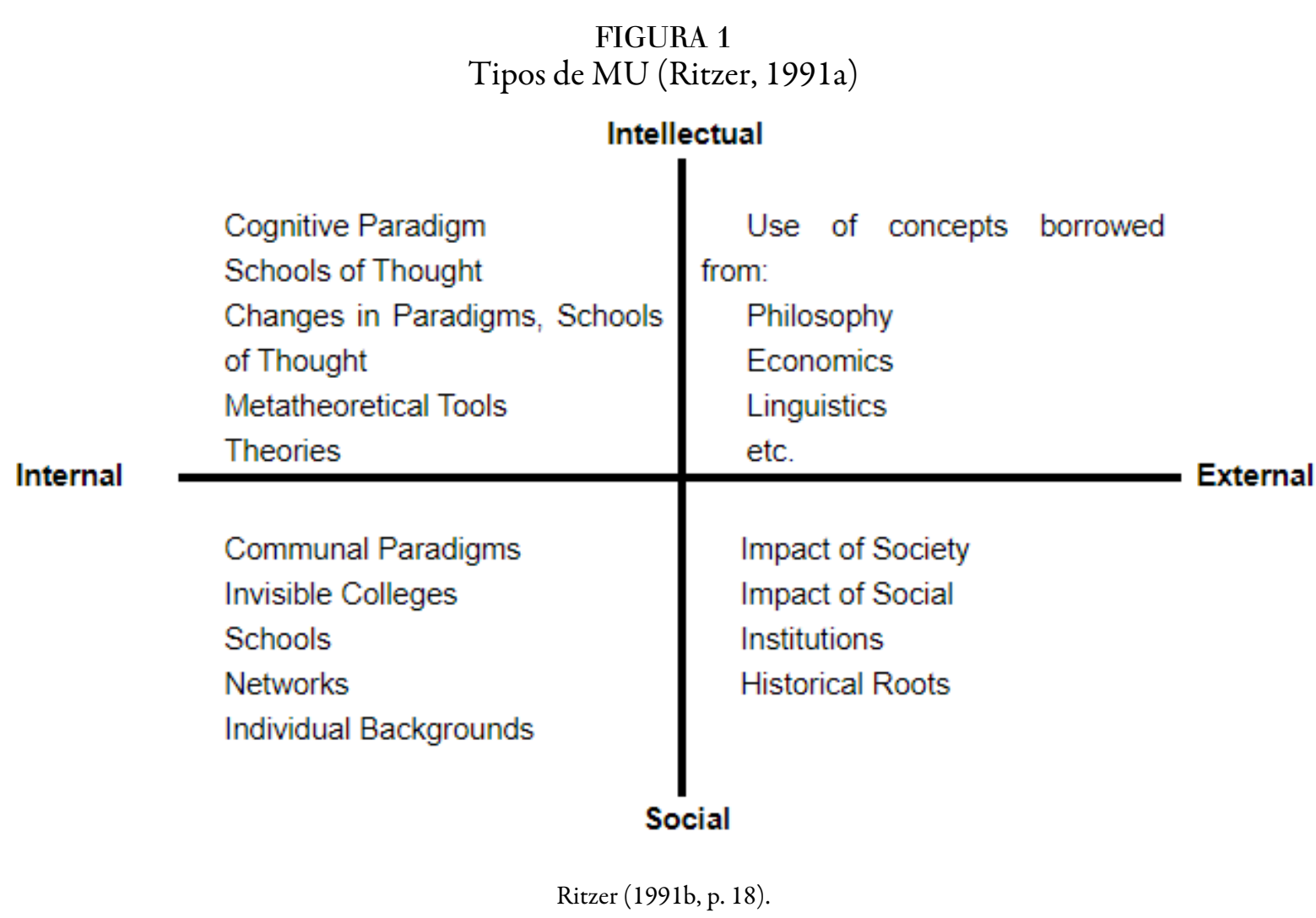

A abordagem intelectual-interna se refere a escolas de pensamentos, paradigmas e teorias em um domínio e sua influência. A abordagem social interna "concentra-se em grupos relativamente pequenos de teóricos que têm ligações diretas entre si”. Envolve o estudo dos teóricos do domínio para identificar paradigmas comunitários, faculdades invisíveis, escolas, redes, origens individuais, etc. (Ritzer, 1991b, p. 19-20, tradução nossa).

Além disso, a abordagem externo-intelectual preocupa-se com ideias, ferramentas, conceitos e teorias de outras disciplinas acadêmicas. E, a abordagem social-externo "envolve a mudança para o nível mais macro para olhar para a sociedade mais ampla e a natureza de seu impacto na teorização sociológica" (Ritzer, 1991b, p. 21, tradução nossa).

Embora metateoria seja um conceito que se originou na sociologia, a metateoria foi utilizada para fins deste estudo. Buscou-se obter uma melhor compreensão do domínio usando $\mathrm{Mu}$, e produzir uma perspectiva que cobre parte ou todo o domínio com Mo, duas das metateorias propostas por Ritzer. Mo é o terceiro tipo de metateoria proposta por Ritzer, ou seja, o estudo da teoria orientado para o objetivo de produzir uma perspectiva que engloba parte ou toda a teoria (Ritzer, 1991b).

Por meio da perspectiva de Rizer (1991b), foi possível propor uma metateoria da epistemologia. de KO. A escolha desse método é justificada por compreender que "a metateoria apresenta todas as situações ou estados de elementos que podem ser expressos dentro das possibilidades lógicas do aparato conceitual de uma teoria". Além disso, "formar uma teoria da unidade a partir de uma metateoria significa a especificação de seus conceitos gerais para se adequar a determinados cenários concretos que o pesquisador desejava estudar" (Vakkari e Kuokkanem, 1997, p. 453). Foi possível perceber que "além da satisfação mental, o trabalho metateórico nos permite representar, organizar e explicar a constituição teórica do campo e, desta forma, recriar o conhecimento" (Arboit, 2014, p. 24).

Os métodos da Teoria Fundamentada em Dados (Grounded Theory), codificação e memorando, também são aplicados na análise do corpus desta pesquisa, que é composto por 31 artigos sobre epistemologia da OC, publicados na revista Knowledge organization. 
Estuda-se nesta pesquisa a epistemologia da OC a partir de uma perspectiva hermenêutica, (Alvesson \& Sköldberg, 2009; Tennis, 2005a), por considerá-la diretamente relacionada à metodologia e à filosofia da ciência; é também uma importante forma de reflexão e um resultado da interpretação. "A hermenêutica como metodologia de interpretação preocupa-se com os problemas que surgem ao lidar com ações humanas significativas e os produtos dessas ações, principalmente os textos” (Mantzavinos, 2016, p. 1).

O compromisso ético desta pesquisa ampara-se na fidelidade às ideias dos autores, expressas nos artigos analisados como parte do corpus desta pesquisa. Dessa forma, pode-se construir um argumento válido e confiável. Uma vez apresentados o compromisso ontológico, ético e a postura epistêmica, apresenta-se na próxima seção como a pesquisa foi conduzida neste artigo.

Uma investigação metateórica segue três etapas para atingir seu objetivo geral: coleta, análise e síntese (Tennis, 2005a). A primeira parte da pesquisa desenvolveu um estudo exploratório baseado na coleta de dados. Em seguida, desenvolve-se a teoria fundamentada para a análise e síntese. Descreve-se nesta seção o estudo exploratório, a análise e a síntese.

\subsection{Estudo exploratório e coleta de dados}

O estudo exploratório apoia a compreensão do domínio. O corpus desta pesquisa é composto por artigos publicados na revista Knowledge organization. O periódico está indexado na base de dados WoS desde 1993 e, a base de dados foi a fonte de informação da qual os artigos foram recuperados. Buscamos artigos publicados de 1993 a 2017 utilizando a seguinte consulta: (epistemolog* OR "theor* of knowledge") AND ("information organization" OR "knowledge organization"). Alguns autores utilizam "teoria do conhecimento" e outros "epistemologia" para se referir ao mesmo conceito, por isso incluímos os dois termos na estratégia de busca. O mesmo acontece com a "organização da informação" e a "organização do conhecimento".

Recupera-se 33 artigos e 2 deles foram excluídos do corpus após análise do título, resumo e palavras-chave. Por fim, o corpus é composto por 31 artigos publicados na revista Knowledge organization até 2017. A coleta de dados foi realizada em 13 de julho de 2018. Os artigos foram coletados no gerenciador de referências Zotero onde são organizados e armazenados.

Os artigos da revista Knowledge organization foram selecionados por ser reconhecida como a principal publicação na área desde a sua primeira edição em 1974, quando o título da revista era International classification (Guimarães, 2014). A revista tornou-se a publicação oficial da ISKO em 1989, conforme descrito no documento do preâmbulo da sociedade (ISKO, 1989, p. 1). Muitos estudos reconheceram a importância do ISKO e que a sociedade expressa um pensamento que traz à tona um novo campo teórico e profissional (Arboit, 2014; Dahlberg, 1995; Sales \& Murguia, 2015).

\section{ANÁLISE}

A teoria fundamentada nos dados é uma metodologia desenvolvida por Glaser e Strauss $(1967$, p. 1) e é "a descoberta da teoria a partir de dados sistematicamente obtidos em pesquisas sociais". O pesquisador lê o texto do corpus em busca de itens de interesse e, a seguir, os codifica. Tomamos emprestado da teoria fundamentada esses procedimentos sistemáticos a fim de identificar, analisar e descrever a concepção de epistemologia em OC. Dois desses procedimentos são a codificação e o memorando e o seu uso nesta pesquisa será descrito nas seções a seguir.

A codificação é um processo de fazer interpretações e envolve a interação com os dados (análise) usando diferentes técnicas. A codificação é um dos procedimentos sistemáticos da teoria fundamentada e nesta 
pesquisa seguimos as três rodadas de codificação (eixo horizontal) propostas por Strauss (1987): codificação aberta, codificação axial e codificação seletiva.

Existem três níveis de codificação. "O nível físico lida com o texto e os códigos embutidos nesses textos durante o processo de codificação. O nível verbal lida com as palavras e frases dos textos. O nível abstrato lida com atributos e concepções", (Tennis, 2005a, p. 47).

O software ATLAS.ti foi utilizado como ferramenta de análise e pesquisa de dados qualitativos para desenvolver a análise e síntese da pesquisa. Cada artigo foi analisado nas três rodadas de codificação. Descrevese o processo de codificação e como a análise foi abordada nesta pesquisa.

a) Codificação aberta: codificaram-se diferentes incidências em tantas categorias quanto possível. Conforme os artigos era analisados, novas categorias surgiram e novos incidentes se encaixaram nas categorias existentes. Por meio desse processo foi possível desenvolver categorias e organizá-las. Ao terminarmos a codificação aberta, foram criados 1.522 códigos.

b) Codificação axial: "consiste em análises intensas feitas em torno de uma categoria de cada vez, em termos dos itens do paradigma (condições, consequências e assim por diante)” (Strauss, 1987, p. 32). É também "um processo para conectar subcategorias a uma categoria". Verificaram-se todos os códigos que foram criados e suas instâncias. Dessa forma, conceitos foram conectados nesta rodada. Dessa forma, eles levaram à criação de categorias principais. Dos 1.522 códigos, reduziu-se para 594 códigos. Como aplicouse a metateoria para melhor compreender a epistemologia da OC, os códigos foram organizados nas quatro abordagens da tabela Mu: interno-intelectual (287 códigos), interno-social (19 códigos), externo-intelectual (270 códigos), externo- social (7 códigos).

c) Codificação seletiva: nesta fase "a codificação axial é continuada em um nível mais profundo de abstração. O objetivo desta rodada é elaborar as categorias centrais nas quais as demais se integrem", (Flick, 2004, p. 194). Nesta rodada, delimita-se a codificação para aquelas variáveis que se relacionam com as variáveis centrais de maneiras suficientemente significativas para ser a base para a análise e síntese. Portanto, são selecionados os códigos considerando as questôes de pesquisa. Como resultado, três famílias de atributos foram criadas a partir da codificação seletiva. As famílias de atributos representam o objeto central do estudo e, são eles: o conceito de epistemologia em OC com 2 atributos; a finalidade da epistemologia da OC com 11 atributos; e as posturas epistêmicas influenciando o domínio da OC com 24 atributos. Neste artigo, apresenta-se a terceira família de atributos, as posturas epistêmicas que influenciam o domínio da OC.

Durante a codificação, consideram-se questões teóricas, hipóteses, resumo de códigos, etc., ou seja "um método de acompanhar os resultados da codificação e estimular a codificação posterior, e também um meio importante para integrar a teoria”. Este processo é chamado de memorando (Strauss 1987, p. 22).

Os memorandos funcionaram como uma ferramenta essencial durante todo o processo de análise e síntese. À medida que a análise e a síntese dos dados foi desenvolvida, os memorandos foram examinados em busca de insights e explicações para as questões abertas durante o processo de criação. Depois de analisar os 31 artigos por meio de diferentes rodadas e níveis de codificação, memorização, conectando diferentes conceitos e categorias centrais, reuniu-se o material para desenvolver uma síntese metateórica. Buscou-se obter um melhor entendimento $(\mathrm{Mu})$ da epistemologia da OC, conforme sugerido por Ritzer (1991). As categorias principais, criadas a partir de codificação e memorando, são analisadas na próxima seção.

\subsection{Síntese metateórica da epistemologia da organização do conhecimento}

Acredita-se, assim como Richard Smiraglia que "um componente muito importante da ciência da OC deve ser a epistemologia, que é a própria ciência do conhecimento (Smiraglia, 2013, p. 3). A forma como as pessoas interpretam os textos a serem organizados, como buscam as informaçóes de que precisam, por exemplo, estão ligados a teorias de interpretação que são epistemologias e ideias que são desenvolvidas histórica, cultural, social e cientificamente. Reconhece-se que a epistemologia influencia a OC como uma 
disciplina, mas também as atividades de OC (classificação, indexação, catalogação, etc.) e a criação e design do Sistema de Organização do Conhecimento (SOC).

Posturas epistêmicas (pragmática, positivista, operacionalista, referencial, instrumental, empirista, racionalista, realista, etc.) levam a afirmações sobre que tipo de conhecimento pode ser criado por meio da pesquisa e como é reunido e apresentado. Por meio de posturas epistêmicas, temos uma visão sistemática da realidade, nosso conhecimento dela e o significado que podemos atribuir a ela (Tennis, 2008, p. 103).

É proposto olhar para a literatura científica da OC publicada na revista Knowledge Organization sobre epistemologia da OC e descrever a influência epistemológica no domínio. A Figura 1 contém a representação da família de atributos "posturas epistêmicas influenciando o domínio da OC".

FIGURA 2

Posições epistêmicas que influenciam o domínio da OC

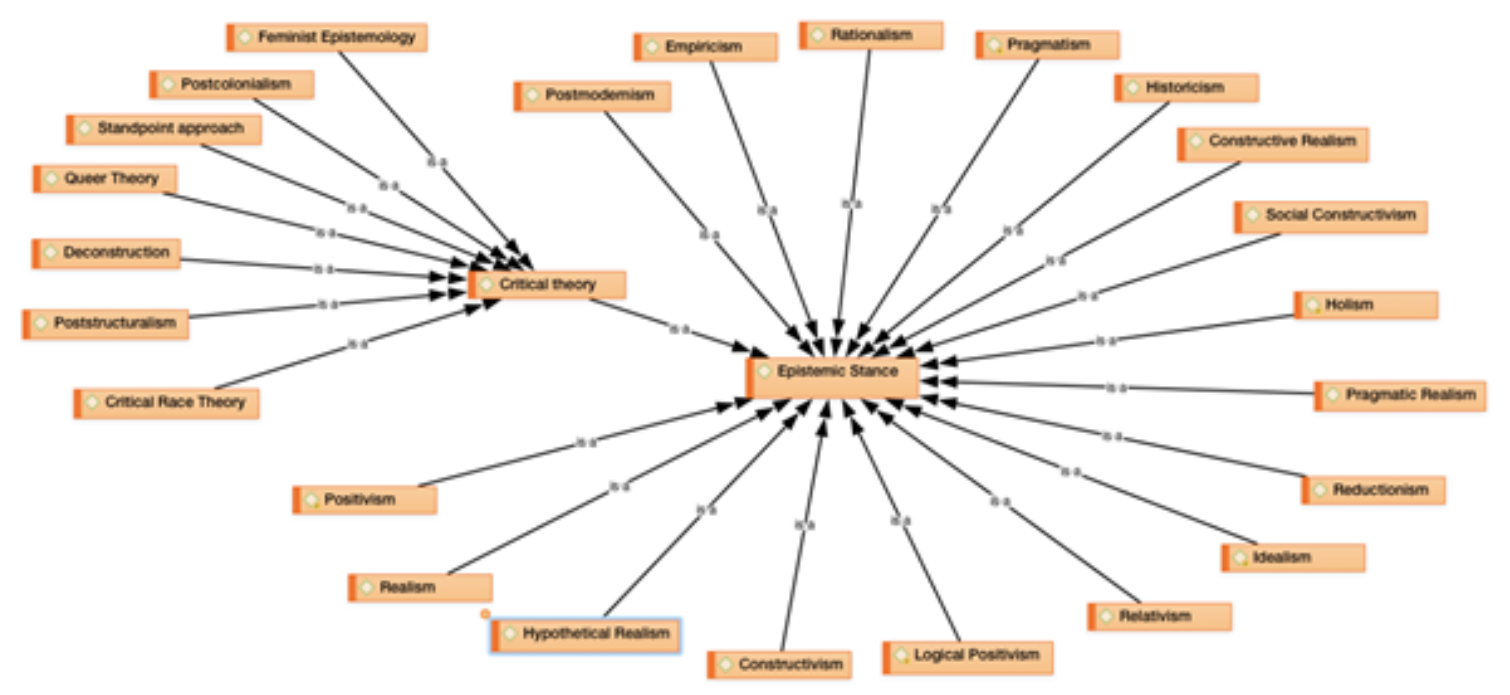

Fonte: dados da pesquisa (2020).

Portanto, apresenta-se uma síntese das posturas epistêmicas que influenciam o pensamento dos autores nas pesquisas que compõem o corpus desta pesquisa. A análise é baseada na classificação de Hjørland de posturas epistêmicas no domínio da OC: empirismo, racionalismo, historicismo e pragmatismo.

O primeiro olhar para o artigo de Garcia Marco e Esteban Navarro (1993, p. 126) mostra uma postura historicista, uma vez que eles refletem sobre os antecedentes da classificação para melhorar e esclarecer as tarefas práticas dos especialistas em informação ou bibliotecários. No entanto, também identificam-se alguns traços do pensamento empirista, pois os autores enfocam as ciências cognitivas e reconhecem que,

[...] compreender a natureza implica coletar experiências por meio de sentidos que posteriormente são processados e formalizados em conceitos e discursos por meio de um processo que inclui, em primeiro lugar, classificar, por meio da distinção entre elementos, agrupá-los por dimensões relevantes e construir critérios de comparação. Em segundo lugar, implica ordenar colocando, conectando e relacionando elementos ao longo de dimensões espaciais, temporais e outras. Em terceiro lugar, consiste em organizar, através do armazenamento, conservação e eliminação de elementos e estabelecer relações de acordo com diferentes critérios e construir um sistema de conhecimento que se torna cada vez mais complexo.

Essa descrição da compreensão da realidade tem características empiristas relacionadas, por exemplo, à crença de que qualquer afirmação de conhecimento genuína pode ser testada pela experiência. A abordagem pragmatista também é revelada por meio do texto. Garcia Marco e Esteban Navarro (1993, p. 130) afirmam que o processo de representação "pode ser formalizado em termos da correspondência entre os elementos perceptivos do ambiente como elementos efetivos do sistema e as interações perceptivas do ambiente como interações efetivas entre elementos do sistema”. 
A abordagem pragmatista é identificada nesse depoimento uma vez que existe a preocupação com a representação da informação e do conhecimento considerando a comunicação efetiva por meio da compreensão dos diferentes pontos de vista. Portanto, o contexto é importante em seu relacionamento com o sistema. Mesmo tendo identificado três diferentes posturas epistêmicas influenciando a pesquisa de Garcia Marco e Esteban Navarro (1993), a postura empirista é predominante no artigo.

A introdução do conceito de imagens da OC por Bies (1996) ao apresentar a importância de metáforas e imagens como "árvore do conhecimento" e "mapa do conhecimento" demonstra a influência da semiótica e mais especificamente do pragmatismo na pesquisa de OC. A abordagem pragmática de Bies (1996, p. 7) considera que "não só o conhecimento como tal, mas também a sua organização, representação, condensação e comunicação são uma parte essencial da memória cultural". Além disso, reconhece que "não só as ideias e os temas, mas também a sua ordenação e organização pertencem ao nosso patrimônio cultural".

A análise de domínio e os três tipos de teorias e conceitos constituídos são abordados por Hjørland e Hartel (2003): teorias ontológicas, teorias epistemológicas e conceitos sociológicos. Eles reconhecem o realismo filosófico e o construtivismo social influenciando a relação entre essas teorias.

Os autores seguem uma abordagem pragmática e historicista em suas pesquisas. Eles acreditam que as dimensões epistemológicas podem ser descobertas pelo estudo dos desenvolvimentos históricos em um domínio e que a classificação e outros processos da OC podem considerar diferentes paradigmas e sua influência na sociedade e em diferentes contextos. Além disso, "à medida que o conhecimento se desenvolve e evolui, a visão das estruturas do mundo e as relações entre os diferentes conceitos mudam simbioticamente" (Hjørland \& Hartel, 2003, p. 244).

Todos os artigos de Hjørland analisados neste artigo expressam uma postura pragmática e/ou historicista (2003, 2008, 2013a, 2014, 2017). Em duas delas ele se aproxima da concepção da OC (Hjørland, 2003, 2008). Hjørland (2003) reconhece que a união básica da OC são relações semânticas entre conceitos. Ele também explica que essas "relações semânticas não podem ser estabelecidas principalmente por suposições universalistas, mas principalmente devem ser entendidas como específicas do domínio, conforme descobertas por (e construídas por) disciplinas científicas” (Hjørland, 2003, p. 107). Essas são suposições feitas com base na teoria do pragmatismo.

Além disso, quando Hjørland propõe um paradigma sociocognitivo baseado em pressupostos epistemológicos, ele também demonstra a influência pragmática. Hjørland (2008, p. 99) afirma que "os domínios são influenciados por visões epistemológicas em mudança e tendências interdisciplinares e, a epistemologia é uma forma simples e mais profunda de compreender a conexão entre a OC e outros domínios, como a linguística". Ele defende que uma metateoria satisfatória para fazer e explicar essas conexões é o pragmatismo. Afirma ainda que o pragmatismo e o historicismo são epistemologias sociais (Hjørland, 2020).

Seguindo o mesmo pensamento, Castanha e Grácio (2014) propõem que a análise de domínio e a metateoria sejam consideradas contribuições significativas para os estudos bibliométricos ao enfatizar a necessidade de análises epistemológicas, sociológicas e históricas, bem como de outras abordagens qualitativas. Mesmo que os estudos bibliométricos sejam, em sua maioria, considerados um método empirista, as autoras acreditam que as análises epistemológica, sociológica e histórica podem ser aplicadas por meio de abordagens de análise de domínio e metateoria ligadas aos estudos bibliométricos, evidenciando a presença de uma postura pragmática.

A suposição de que o conhecimento é falível e a OC tem que considerar diferentes teorias/visões, seus fundamentos e diferentes epistemologias (por exemplo, empirismo, racionalismo, historicismo ou pragmatismo), também torna evidente a postura pragmática sobre os pensamentos de Hjørland. (Hjørland, 2013a, 2014, 2020).

Smiraglia (2015) considera que há um domínio vibrante em torno da análise de domínio para a OC. O discurso dominante entre o grupo que está aplicando a análise de domínio está entre a necessidade pragmática de um SOC específico para diferentes domínios e as posições ontológicas e epistemológicas clássicas em OC 
representadas pela teoria do conceito. Pode-se considerar que a postura pragmática de Smiraglia (2015, p. 610) pode ser identificada em suposições como: "a análise de domínio para OC é um campo muito vibrante de pesquisa e desenvolvimento, não apenas para OC como ciência, mas para a humanidade em geral".

Ao seguir a identificação das posturas epistêmicas dos autores, afirma-se que em ambas as pesquisas de Tênis (2005b, 2008), encontramos uma abordagem pragmática. Como Tennis (2005b, p. 81) afirma que a abordagem experiencialista das estruturas classificatórias "busca construir uma estrutura classificatória multidimensional que dê conta das interseções do significado individual e social, e uma interseção das estruturas formais e associativas", por exemplo, é fácil identificar duas características do pragmatismo, a abordagem socialmente inserida e pluralista.

O artigo de Moura (2014, p. 304) é baseado na formação discursiva de Foucault, e há conexão com a abordagem sócio-cognitiva da OC. O artigo teve como objetivo "identificar as oportunidades e desafios de incorporar considerações epistemológicas no ato de adquirir conhecimento na consolidação da OC, processos e dispositivos de mediação na emergência dos fenômenos". A autora afirma que o estudo realizado permitiu observar um esforço coletivo para a resolução de um modelo de interoperabilidade semântica para a rotulagem de conteúdos com base nas melhores práticas de descrição dos objetos partilhados em Espaços de Informação Semântica Social (SSIS).

O artigo traz à luz a importância das abordagens colaborativas no domínio da OC. A autora afirma que os espaços de informação semântica social representam boas perspectivas de consolidação de procedimentos que podem representar dinamicamente o conhecimento emergente compartilhado em uma rede. Podese considerar que "esta abordagem teórica e tecnológica pode ajudar a aprimorar ferramentas semânticas, classificações, taxonomias de navegação e metodologias para a construção de uma linguagem de indexação em ambientes digitais colaborativos" (Moura, 2014, pp. 309-310).

Gnoli (2012) questiona o tipo tradicional de SOC baseado em disciplinas acadêmicas. Ele apresenta as três dimensões da OC e as diferencia: ôntica, epistêmica e documental. Portanto, o autor reconhece que é necessário distinguir entre as diferentes dimensões dos itens de conhecimento e tratar cada dimensão separadamente de forma adequada.

Muitas características pragmáticas são percebidas no pressuposto de Gnoli (2012) em relação ao Manifesto de León (ISKO, 2007), quais sejam: - há uma tendência frequente para uma crescente interdisciplinaridade do conhecimento que exige essencialmente novos SOCs e esta inovação é viável; - em vez de disciplinas, as unidades básicas do novo sistema devem ser fenômenos; - o novo SOC deve permitir que os usuários mudem de uma perspectiva ou ponto de vista para outro; - as conexões podem ser expressas e gerenciadas por técnicas analítico-sintéticas.

Há um interesse crescente na natureza dependente do contexto do conhecimento humano. E, da mesma forma que Gnoli (2012), outros autores buscam a integração entre as teorias ontológicas e epistemológicas. Kleineberg (2013) propõe uma abordagem integrativa que se poderia rotular de "classificação-comoontologia/epistemologia”, o que denomina realismo construtivo. Essa abordagem é baseada em um conceito de fenômeno triádico e em três princípios organizadores fundamentais: os "níveis de ser" (ontologia), os "níveis de conhecimento" (epistemologia) e o "pluralismo metodológico integral” (metodologia). Kleineberg (2013) busca evitar a falácia comum de que pluralismo epistêmico implica relativismo epistêmico.

A suposição de Kleineberg (2013) de que o conhecimento humano é sempre conhecimento em contexto e uma organização sistemática de contextos epistêmicos é obrigatória para a teoria da OC, em particular, para qualquer abordagem baseada em fenômenos, também revela seu pensamento pragmático. Da mesma forma que Kleineberg (2013), Ridi (2016) defende a postura do realismo construtivo, buscando uma teoria de síntese entre objetivismo e subjetivismo. A teoria consiste em reconhecer que a realidade não é completamente dada nem completamente construída e constitui o paradigma dominante tanto na epistemologia quanto nos estudos da OC. 
Complementarmente a essa abordagem, encontra-se a pesquisa de Hansson (2013) ao discutir a relação entre epistemologia, organização social e OC. Ele assume uma perspectiva de análise materialista no sentido neomarxista. $\mathrm{O}$ autor acredita que "as relações entre estrutura social, poder econômico e divisão do trabalho regem a forma como são feitas as reivindicações de legitimidade epistemológica para os sistemas de organização do conhecimento" (Hansson, 2013, p. 385). Entre suas análises e proposições, Hansson (2013) afirma que a sociedade é a unidade básica da OC, o que demonstra uma perspectiva pragmática.

Santis e Souza (2014) também abordam a representação da música, e discutem sobre a construção de um fundamento epistemológico utilizado especificamente para classificar a canção popular. Uma vez que as mudanças comerciais influenciam a forma como a música é representada, os autores discutem a marcação colaborativa como uma alternativa. Hansson (2013) também propõe a prática da marcação social e folksonomias na sociedade contem porânea como exemplos para mostrar como a relação entre epistemologia, organização social e OC tem se mostrado historicamente estável.

Portanto, Santis e Souza (2014) reconhecem que a semiótica estabelece critérios para estudar o pensamento complexo. E, do ponto de vista semiótico, considera que a influência da visão fenomenológica leva à necessidade de uma abordagem pragmática da classificação artística.

A postura pragmática também está presente no artigo de Marteleto e Carvalho (2015), que reuniu construções teóricas e metodológicas desenvolvidas por Birger Hjørland e Pierre Bourdieu para investigar as estruturas de produção, organização e comunicação do conhecimento de um ponto de vista crítico, com foco em saúde.

A autoria está relacionada à filosofia e à OC na pesquisa de Silveira e Saldanha (2016). Do seu ponto de vista, "os estudos que integram questóes filosóficas, conceituais e culturais na representação documental permitem uma reflexão crítica sobre o desdobramento do uso da autoria nas práticas informacionais", (Silveira e Saldanha, 2016, p. 265).

A abordagem pragmática de Silveira e Saldanha (2016) considera que o debate sobre o "próprio nome" carece da atenção crítica da OC, responsável por pensar e implementar formas de identificar, ordenar e acessar conteúdos e continentes, atividades que ora reconhecem, ora instituem, ora apagam o "nome próprio" como elemento sociocultural, anterior a um "ponto de acesso".

A pesquisa de Martínez-Ávila e Beak (2016) estudou os métodos e referenciais teóricos que analisam a pesquisa de Hope Olson. Os autores afirmam que sua análise se baseia na postura pós-estruturalista adotada por Hope Olson ao longo de sua carreira. Afirmamos que os autores têm uma posição crítica para interpretar os métodos, teorias e epistemologias presentes nas pesquisas de Olson, que evidenciam a visão pragmatista sobre os argumentos dos autores.

Também identificamos as implicações do big data para a OC, abordadas por Ibekwe-SanJuan e Bowker (2017). Os autores reconhecem a necessidade de SOCs construídos humanamente e confrontam os debates dentro da comunidade OC sobre a relevância das classificações bibliográficas universais e do tesauro na web com as discussões em curso sobre os pressupostos epistemológicos e metodológicos subjacentes à investigação baseada em dados.

Eles propõem que o desafio para a OC é, portanto, "reinventar-se em um ecossistema de informações repleto de algoritmos que processam dados continuamente e fornecem conteúdo digital adaptado aos perfis dos usuários, em vez de se concentrar em um bases de conhecimento de tamanho adequado a todas construídas a priori” Ibekwe-SanJuan e Bowker (2017, p. 196). É proposta a integração de SOCs tradicionais com sistemas participativos e/ou colaborativos, determinando como ambas as abordagens podem ser combinadas na concepção de SOCs para aplicações específicas e categorias de usuários. Olhando para as proposições apresentadas por Ibekwe-SanJuan e Bowker (2017), identifican-se algumas características pragmatistas: contingente, socialmente inserido e pluralista.

Ainda seguindo o pensamento pragmático, Martínez-Ávila, Semidão e Ferreira (2016) analisam a configuração metodológica de teorias críticas no domínio da OC. Eles também criticam a neutralidade 
nos processos da OC e o design de SOCs universais. Apresentam teorias críticas como uma resposta aos problemas éticos que afetam grupos específicos nesses sistemas. Para eles, a teoria crítica influencia as "esferas epistemológica, conceitual, metodológica, axiológica e até retórica” (Martínez-Ávila, Semidão \& Ferreira, 2016, p. 118).

A pesquisa de Martínez-Ávila, Semidão e Ferreira (2016) é muito importante para o campo, pois reconhece-se a forte presença de estudos de teoria crítica no domínio da OC. Neste estudo, Campbell (2000), Furner (2009), Samuelsson (2010), Martínez-Ávila, Semidão e Ferreira (2016) mostram diferentes abordagens que são relevantes para os estudos e prática da OC.

Campbell (2000) aborda a distinção teórica entre os conceitos de aboutness e significado na OC relacionados aos estudos homossexuais. À medida que o artigo explora o debate literário e suas implicações para o projeto de sistemas de acesso de sujeitos para comunidades gays e lésbicas, Campbell reconhece que os designers de sistemas de acesso de sujeitos podem esperar trabalhar em um contexto de intenso escrutínio e persistente controvérsia.

O autor reconhece as atividades da OC como um processo subjetivo, identifica-se a abordagem pragmatista ao longo do texto, o que se justifica ao considerar que a teoria queer e todas as teorias críticas estão relacionadas ao pragmatismo. Ao abordar a teoria da classificação e o design de SOCs, o autor sempre se refere a sistemas e processos contextuais, socialmente determinados e culturalmente relativos.

Quando Furner (2009) pergunta "quão bem os sistemas de OC representam a identidade" evidencia-se uma das preocupações no domínio da OC, a representação do conhecimento da identidade racial, étnica, de gênero, sexual, nacional, linguística e religiosa. Aproximando-se de uma postura pragmática, Furner (2009, p. 14) propõe que "queremos que nossos sistemas de OC representem todas as identidades-como-sujeitos de uma maneira justa que respeite os direitos de todos". Portanto, essa abordagem combina as premissas de diferentes teorias críticas buscando melhor representar a identidade nos SOCs.

A epistemologia feminista é abordada por Samuelsson (2010). A autora afirma que sua abordagem teóricometodológica é pós-estruturalista e orientada para o discurso. Defende uma perspectiva construcionista social e antiessencialista. Além disso, à medida que analisa os SOCs que indexam e classificam os textos de pesquisa feministas em um contexto bibliográfico sueco, identifica-se uma pesquisa preocupada com a $\mathrm{OC}$ da pesquisa feminista.

Identificam-se os traços da postura que Samuelsson (2010, p. 9) assume em sua pesquisa na argumentação sobre os SOCs universais. Ela afirma que "o conhecimento feminista é marginalizado e tornado invisível pelos sistemas de organização do conhecimento gerais. Esta marginalização pode, por sua vez, ser interpretada como consequência de uma suposta epistemologia e ontologia objetivista e universalista incorporada nesses sistemas".

Outra postura vibrante em OC é o historicismo, que se aproxima muito do pragmatismo. Já foram citados Garcia Marco e Esteban Navarro (1993), Hjørland e Hartel (2003) e Hjørland (2003, 2008, 2013b, 2014, 2017) como influenciados pela postura historicista. Entretanto, outros artigos também apresentam as características dessa postura.

Gnoli (2008) teve a tarefa de abordar dez questões básicas no século 21 a respeito do domínio da OC. Sua abordagem baseou-se na literatura e na reflexão disponíveis atualmente, algumas questões relevantes que parecem mais gerais e de interesse de longo alcance. Reconhece-se a postura historicista na abordagem de Gnoli (2008), uma vez que ele delineia ao longo do tempo as expectativas para o futuro do domínio, considerando o contexto e, principalmente, os estudos epistemológicos. Encontra-se também a postura historicista na pesquisa de Dousa (2010) sobre o pragmatismo clássico e suas variedades, considerando que o autor olha para trás para a história da OC para traçar as características de diferentes abordagens do pragmatismo no domínio da OC.

Outra abordagem historicista é identificada na proposição de Saldanha (2014) de um estudo históricoepistemológico da OC com foco na década de 1930. Ele desenvolve um pensamento orientado à pragmática 
da linguagem na cena filosófica do período. Há também a influência do pragmatismo em seu pensamento, o que está presente, por exemplo, em sua afirmação: "demonstramos como a pragmática, hoje um discurso comum na epistemologia da ciência da informação, pode receber considerações oriundas de outras demarcações do nosso pensamento, evidenciando a necessidade de uma revisão filosófica da OC" (Saldanha, 2014, p. 302).

Também encontra-se a postura idealista representada na pesquisa de Kiel (1994), quem contradiz a diferenciação de conhecimento de Jaenecke como: conhecimento central, conhecimento periférico e pseudo conhecimento. Ele explica que as abordagens de Jaenecke são realistas e, por outro lado, afirma que "o conhecimento não pode ser separado da subjetividade individual ou cultural". Kiel (1994, p. 151) reconhece sua postura como idealista. "Na estrutura do idealista, propriedades, entidades e relações não existem independentemente de nossa mente ou de nossa capacidade de raciocinar. Portanto, a observação é considerada dependente de nossa mente".

Por fim, descreve-se a postura empirista e racionalista de Zins (2004). O autor considera que "o conhecimento como estado de espírito é produto de uma síntese”. Ele reconhece que qualquer percepção empírica é o produto da síntese de uma multiplicidade de dados sensoriais. Zins (2004, p. 54) reconhece sua postura racionalista, e identifica "em qualquer percepção a priori componentes, que dão sentido à diversificada matéria-prima sensorial e a constrói em uma unidade.

Ao longo da análise dos estudos, reconhecemos que o pensamento pragmático está presente na maioria dos estudos. A postura pragmática é sublinhada pelos argumentos relativos à integração de abordagens epistemológicas, metodológicas, sociológicas e históricas para melhorar o processo e os sistemas no domínio da OC. Há também o estímulo para repensar o desenho de sistemas universais em OC e dar como certo o ambiente social e cultural e sua influência no processo e nas ferramentas da OC. A próxima seção contém as considerações finais da pesquisa.

\section{CONSIDERAÇÕES FINAIS}

Os estudos epistemológicos e a influência da epistemologia no domínio da OC são reconhecidos pela comunidade de OC. No entanto, o domínio carece de estudos sobre o assunto. Entende-se que a epistemologia tem um papel importante no que diz respeito à questão teórica e metodológica tanto na pesquisa em OC quanto no desenvolvimento de SOCs.

Este artigo apresentou um estudo metateórico de 31 artigos escritos por 32 pensadores da temática de epistemologia da OC. Objetivou-se descrever a concepção de epistemologia no periódico Knowledge organization por meio da metateoria. $\mathrm{O}$ primeiro objetivo foi analisar a produção científica sobre epistemologia da OC, publicada na revista. Para atingir esse objetivo fez-se um estudo exploratório, o processo de codificação e memorando, descritos na metodologia.

A análise das posturas epistêmicas mostra o destaque do pensamento pragmatista no domínio, seguido das teorias críticas e do historicismo. Explica-se esta constatação por reconhecer que o paradigma sociocognitivo como dominante na OC. Conhecimento e OC lidam com necessidades e interesses humanos. Além disso, o conhecimento é construído em vários cenários e leva a diferentes reivindicações de conhecimento. Entendese que a pesquisa e a prática da OC tendem ao modelo socialmente pluralista, o que pode estar relacionado ao pensamento pós-moderno sobre a relação entre as pessoas, o conhecimento como construção social e a influência de várias perspectivas.

A partir dos pontos de vista e pensamentos mais expressivos apresentados nos artigos do corpus deste artigo, é possível ter uma perspectiva do domínio da OC. Percebeu-se em diferentes estudos a preocupação com: a) o reconhecimento da importância da conexão entre as abordagens epistemológica, teórica, metodológica, sociológica e histórica, uma vez que constituem a força motriz que delineia a argumentação na obra conceitual da OC; b) a influência das diversas posturas epistêmicas na concepção e 
atualização do SOC; c) a necessidade de representação da identidade no SOC, uma vez que são culturalmente embasadas e enviesadas; d) a crítica aos sistemas universais e os argumentos sobre os sistemas neutros e preconceituosos, objetivismo x subjetivismo, considerando o acesso inadequado a grupos marginalizados; e) os pontos de vista objetivista e subjetivista são abordados especialmente quando se trata de processos de OC como classificação e indexação. Essas duas ações não são atividades neutras ou objetivas; f) a abordagem analítica de domínio é um paradigma forte no domínio da OC com uma forte influência pragmatista e historicista. Além disso, o desenvolvimento da análise de domínio levantou a questão sobre a subjetividade e objetividade da OC de forma sistemática, uma vez que a análise de domínio carrega em sua natureza um ponto de vista sociológico e epistemológico; g) a presença de estudos abordando teorias críticas para delinear a pesquisa e a prática em $\mathrm{OC} ; \mathrm{h}$ ) a reivindicação da construção de $\mathrm{SOC}$ de domínios específicos, uma vez que os sistemas universais de que dispomos são deficientes e sua remodelação não seria suficiente.

Todas essas preocupações são influenciadas por posturas epistêmicas. Entende-se que não se pode conectar cada pesquisa ou pensamento do autor a apenas uma postura epistêmica específica. Este estudo buscou reconhecer as influências de diferentes posturas epistêmicas ao longo do tempo e da história da OC para compreender o processo de OC e a pesquisa considerando o corpus escolhido para este estudo. Não se afirma que uma epistemologia é melhor ou mais eficiente do que outra. Acredita-se que o processo, SOC e atividades no domínio da OC exigem diferentes abordagens, teorias, metodologias e epistemologias porque se complementam.

Como observação final, afirma-se que a concepção de epistemologia no domínio da OC é o estudo crítico dos princípios, hipóteses e produção de conhecimento no domínio. A epistemologia se preocupa com o conhecimento científico produzido pelo domínio da OC, bem como com a aplicação desse conhecimento para projetar os SOCs e apoiar o processo de OC.

\section{Agradecimentos}

Esta pesquisa foi financiada pela Fulbright Brasil e pela Coordenação de Aperfeiçoamento de Pessoal de Nível Superior (CAPES)

\section{REFERÊNCIAS}

Alvesson, M. \& Sköldberg, K. (2009). Reflexive methodology: new vistas for qualitative research. Thousand Oaks: SAGE. Arboit, A. E. (2014). O processo de institucionalização sociocognitiva do dominio de Organização do Conhecimento a partir dos trabalhos cientificos dos congressos da ISKO [Tese (doutorado), Universidade Estadual Paulista Júlio de Mesquita Filho], Marilia. Recuperado de https://repositorio.unesp.br/handle/11449/123389

Bies, W. (1996). Thinking with the help of images: On the metaphors of knowledge organization. Knowledge organization, 23(1), 3-8. https://doi.org/10.5771/0943-7444-1996-1-3.

Bufrem, L. S. (2012). A prática da pesquisa e os quadros teóricos concorrentes no campo de produção científica em ciência da informação [Proposta de pesquisa apresentada como requisito parcial à renovação e/ou progressão da Bolsa de Produtividade em Pesquisa do Conselho Nacional de Desenvolvimento Científico e Tecnológico (CNPq).].

Bullock, A (Ed.) (1989). Fontana dictionary of modern thought. London: Fontana Press.

Campbell, G. (2000). Queer theory and the creation of contextual subject access tools for gay and lesbian communities. Knowledge organization, 27(3), 122-131. https://doi.org/0.5771/0943-7444-2000-3-122

Castanha, R. C. G. \& Gracio, M. C. C. (2014). Bibliometrics contribution to the metatheoretical and domain analysis studies. Knowledge organization, 41(2), 171-174. https://doi.org/10.5771/0943-7444-2014-2-171

Dahlberg, I. (1995). Current trends in knowledge organization. In F. J. García Marco (Org.). Organización del conocimiento em sistemas de información y documentación (pp. 7-25). Zaragoza: Universidad de Zaragoza. 
Dousa, T. M. (2010). Classical pragmatism and its varieties: on a pluriform metatheoretical perspective for knowledge organization. Knowledge organization, 37(1), 65-71. https://doi.org/10.5771/0943-7444-2010-1-65

Flick, U. (2004). Uma introdução à pesquisa qualitativa (S. Netz, Trad.; 2.ed.). New York: Bookman.

Furner, J. (2009). Interrogating "Identity": A philosophical approach to an enduring issue in knowledge organization. Knowledge organization, 36(1), 3-16. https://doi.org/10.5771/0943-7444-2009-1-3

Garcia Marco, F. J. \& Estebán Navarro, M. A. (1993). On some contributions of the cognitive sciences and epistemology to a theory of classification. Knowledge organization, 20(3), 126-132. https://doi.org/10.5771/0 943-7444-1993-3-126

Glaser, B. G. \& Strauss, A. L. (1967). The discovery of grounded theory: strategies for qualitative research (4. paperback printing). New York: Aldine.

Gnoli, C. (2008). Ten long-term research questions in knowledge organization. Knowledge organization, 35(2-3), 137-149. https://doi.org/10.5771/0943-7444-2008-2-3-137

Gnoli, C. (2012). Metadata about what? Distinguishing between ontic, epistemic, and documental dimensions in knowledge organization. Knowledge organization, 39(4), 268-275. https://doi.org/10.5771/0943-7444-20124-268

Guimarães, J. A. C. (2014). Análise de domínio como perspectiva metodológica em organização da informação. Ciência da informação, 43(1), 13-21. Recuperado de http://revista.ibict.br/ciinf/article/view/1415/1593

Hansson, J. (2013). The materiality of knowledge organization: Epistemology, metaphors and society. Knowledge organization, 40(6), 384-391. https://doi.org/10.5771/0943-7444-2013-6-384

Hjørland, B. (2002a). Domain analysis in information science: eleven approaches: traditional as well as innovative. Journal of documentation, 58(4), 422-462. https://doi.org/10.1108/00220410210431136

Hjørland, B. (2002b). Epistemology and the socio-cognitive perspective in information science. Journal of the American Society for Information Science and Technology, 53(4), 257-270. https://doi.org/10.1002/asi.10042

Hjørland, B. (2003). Fundamentals of knowledge organization. Knowledge Organization, 30(2), 87-111. https://do i.org/10.5771/0943-7444-2003-2-87

Hjørland, B. (2005). Empiricism, rationalism and positivism in library and information science. Journal of documentation, 61(1), 130-155. https://doi.org/10.1108/00220410510578050

Hjørland, B. (2008). What is knowledge organization (KO)? Knowledge organization, 35(2-3), 86-101. https://doi .org/10.5771/0943-7444-2008-2-3-86

Hjørland, B. (2013a). Theories of knowledge organization: theories of knowledge. Knowledge organization, 40(3), 169-181. Library \& information science abstracts (LISA). Recuperado de http://search.proquest.com/docvie $\mathrm{w} / 1692278816$ ? accountid $=14784$

Hjørland, B. (2013b). Theories of knowledge organization: Theories of knowledge. Knowledge organization, 40(3), 169-181. Library \& information science abstracts (LISA). http://search.proquest.com/docview/1692278816 ?accountid $=14784$

Hjørland, B. (2014). Is facet analysis based on rationalism? A discussion of Satija (1992), Tennis (2008), Herre (2013), Mazzocchi (2013b), and Dousa \& Ibekwe-SanJuan (2014). Knowledge organization, 41(5), 369-376. https:// doi.org/10.5771/0943-7444-2014-5-369.

Hjørland, B. (2017). Domain analysis. Knowledge organization, 44(6), 436-464. https://doi.org/10.5771/0943-744 4-2017-6-436

Hjørland, B. (2020). Political versus apolitical epistemologies in knowledge organization. Knowledge organization, 47(6), 461-485. https://doi.org/10.5771/0943-7444-2020-6-461

Hjørland, B. \& Hartel, J. (2003). Afterword: ontological, epistemological and sociological dimensions of domains. Knowledge organization, 30(3-4), 239-245. https://doi.org/10.5771/0943-7444-2003-3-4-239

Ibekwe-SanJuan, F. \& Bowker, G. C. (2017). Implications of big data for knowledge organization. Knowledge organization, 44(3), 187-198. 
ISKO. International Society for Knowledge Organization. (1989). Charter and preamble. Recuperado de http://ww w.isko.org/charter.pdf

ISKO. (2007). VII Congreso ISKO, León, España. Recuperado de http://www.iskoiberico.org/congresos/leon-2007/

Kiel, E. (1994). Knowledge organization needs epistemological openness: A reply. Knowledge Organization, 21(3), 148-152. https://doi.org/10.5771/0943-7444-1994-3-148

Kleineberg, M. (2013). The blind men and the elephant: towards an organization of epistemic contexts. Knowledge organization, 40(5), 340-362. https://doi.org/10.5771/0943-7444-2013-5-340

López-Huertas, M. J. (2015). Domain analysis for interdisciplinary knowledge domains. Knowledge organization, 42(8), 570-580. https://doi.org/10.5771/0943-7444-2015-8-570

Mantzavinos, C. (2016). Hermeneutics. In E. N. Zalta (Org.), The Stanford Encyclopedia of Philosophy (Winter 2016). Metaphysics Research Lab, Stanford University. Recuperado de https://plato.stanford.edu/archives/win2016/ entries/hermeneutics/

Marteleto, R. M. \& Carvalho, L. dos S. (2015). Health as a knowledge domain and social field: dialogues with Birger Hjorland and Pierre Bourdieu. Knowledge organization, 42(8), 581-590. https://doi.org/10.5771/0943-7444 $-2015-8-581$

Martínez-Ávila, D. \& Beak, J. (2016). Methods, theoretical frameworks and hope for knowledge organization. Knowledge organization, 43(5), 358-366. https://doi.org/10.5771/0943-7444-2016-5-358

Martinez-Avila, D., Semidao, R. \& Ferreira, M. (2016). Methodological aspects of critical theories in knowledge organization. Knowledge organization, 43(2), 118-125. https://doi.org/10.5771/0943-7444-2016-2-118

Moura, M. A. (2014). Emerging discursive formations, folksonomy and social semantic information spaces (SSIS): The contributions of the theory of integrative levels in the studies carried out by the Classification Research Group (CRG). Knowledge organization, 41(4), 304-310. https://doi.org/10.5771/0943-7444-2014-4-304

Ridi, R. (2016). Phenomena or noumena?: objective and subjective aspects in knowledge organization. Knowledge organization, 43(4), 239-253. https://doi.org/10.5771/0943-7444-2016-4-239

Ritzer, G. (1991a). Reflections on the rise of metatheorizing in sociology. Sociological perspectives, 34(3), 237-248. h ttps://doi.org/10.2307/1389509

Ritzer, G. (1991b). Metatheorizing in sociology. Lexington, Mass.: Lexington Books.

Ritzer, G. (2001). Metatheorizing in Sociology. In G. Ritzer. Explorations in social theory: from metatheorizing to rationalization (pp. 13-33). Thousand Oaks: SAGE Publications.

Saldanha, G. S. (2014). The philosophy of language and knowledge organization in the 1930s: Pragmatics of Wittgenstein and Ranganathan. Knowledge organization, 41(4), 296-303. https://doi.org/10.5771/0943-744 4-2014-4-296

Sales, R. de \& Murguia, E. I. (2017). Instaurações discursivas da organização do conhecimento: H. E. Bliss e a International Society for Knowledge Organization (ISKO). Ciência da informação, 44(3). https://doi.org/10. 18225/ci.inf..v44i3.1949

Samuelsson, J. (2010). Knowledge organization for feminism and feminist research: a discourse oriented study of systematic outlines, logical structure, semantics and the process of indexing. Knowledge organization, 37(1), 328. https://doi.org/10.5771/0943-7444-2010-1-3

Santis, R. de \& Souza, R. F. de. (2014). Classifying popular songs: possibilities and challenges. Knowledge organization, 41(2), 181-187. https://doi.org/10.5771/0943-7444-2014-2-181

Silveira, N. C. \& Saldanha, G. S. (2016). "Own name" in knowledge organization epistemology: A philosophicaltheoretical debate. Knowledge organization, 43(4), 265-278. https://doi.org/10.5771/0943-7444-2016-4-265

Smiraglia, R. P. (2013). The epistemological dimension of knowledge organization. IRIS - Revista de informação, memória e tecnologia, 2(1), 2-11. Recuperado de https://periodicos.ufpe.br/revistas/IRIS/article/view/498

Smiraglia, R. P. (2015). Domain analysis of domain analysis for knowledge organization: Observations on an emergent methodological cluster. Knowledge organization, 42(8), 602-611. https://doi.org/10.5771/0943-7444-2015-8 $-602$. 
Strauss, A. L. (1987). Qualitative analysis for social scientists. London: Cambridge University Press.

Tennis, J. T. (2005a). Experientialist epistemology and classification theory: Embodied and dimensional classification. Knowledge organization, 32(2), 79-92. https://doi.org/10.5771/0943-7444-2005-2-79

Tennis, J. T. (2005b). Conceptions of subject analysis: a metatheoretical investigation. Recuperado de http://search.pr oquest.com/docview/305423788/

Tennis, J. T. (2008). Epistemology, theory, and methodology in knowledge organization: Toward a classification, metatheory, and research framework. Knowledge organization, 35(2-3), 102-112. https://doi.org/10.5771/09 43-7444-2008-2-3-102

Tennis, J. T. (2015). Foundational, first-order, and second-order classification theory. Knowledge organization, 42(4), 244-249. https://doi.org/10.5771/0943-7444-2015-4-244

Vakkari, P. \& Kuokkanem, M. (1997). Theory growth in information science: applications of the theory of science to a theory of information seeking. Journal of documentation, 53(5), 497-519. https://doi.org/10.1108/EUM 0000000007210

Vickery, B. (1998). Metatheory and information science. Journal of documentation, 53(5), 457-76. https://doi.org/ 10.1108/EUM0000000007206

Zins, C. (2004). Knowledge organization: An epistemological perspective. Knowledge organization, 31(1), 49-54. ht tps://doi.org/10.5771/0943-7444-2004-1-49 